\title{
THE PHYSICAL DEGREES OF FREEDOM IN HOT QUENCHED QCD
}

\author{
Sourendu Gupta \\ Theory Division, CERN, CH-1211, Geneva 23, Switzerland.
}

\begin{abstract}
We demonstrate the absence of a low-lying bosonic pole in the spectral function for the pseudoscalar meson channel for $T>T_{c}$. We also extract a set of effective 4 -fermi couplings and show that this is small in the vector channel for all $T>T_{c}$, but not in the pseudovector channel. A perturbative estimate is compatible with this result. The computations have been performed in quenched QCD on lattices with $N_{\tau}=4$.
\end{abstract}

CERN-TH.6509/92

June 1992 
Finite-temperature QCD computations on the lattice are, in principle, capable of yielding information on real-time processes in a quark-gluon plasma near equilibrium. The general principles are well-known- one measures matrix elements of well-defined operators over the thermal equilibrium ensemble on Euclidean lattices, and performs an analytic continuation into real time. However, such a continuation requires data from lattices with small spacing, and a knowledge of the singularities and discontinuities of the spectral function. Progress on the first point is continuous. Attention has now turned to the second.

It has been known for a long time that the physics of the hot phase of QCD is not trivial; different phenomena can be expected to hold sway at different length scales [1]. Whereas at scales of $1 / T$, and up to $1 / g T$, perturbative accounts of interactions are likely to capture most of the physics, they must break down at scales of $1 / g^{2} T$. This is related to the persistence of an area law behaviour for spatial Wilson loops. At length scales of $1 / g^{3} T$ hydrodynamic modes also become important. In order to understand the physics of this phase, it becomes necessary to identify all physical degrees of freedom, i.e., the collective excitations and quasiparticles of the theory.

A variety of lattice measurements have shown that, for $T \gtrsim 1.5 T_{c}$, interactions in the plasma seem to be weak. Since most lattice computations have been performed with system volumes of $2-3 \mathrm{fm}$, this is in agreement with expectations ( $T$ is of the order of $\left.1 \mathrm{fm}^{-1}\right)$. Nevertheless, very similar measurements carried out at lower temperatures, just above $T_{c}$, also give evidence of large residual interactions. These appear in measurements of energy densities and pressure [2], hadronic screening masses [3,4] and transverse correlators (sometimes called "wavefunctions") [5], and the quark propagator [6]. The main characteristic of these observations is that large effects are visible in low-momentum modes, and only in a few spin channels. We study these interactions in detail.

The immediate problem we address is that of mesonic screening masses. It was recently demonstrated [4] that the baryon and vector/pseudovector meson screening masses $(\mu)$ are equal to thrice and twice the Matsubara frequency, respectively. This verifies that the screening arises from fermionic intermediate states. The plasma, therefore, contains quarks as physical states, in agreement with measurements of the quark number susceptibility [7]. However, scalar and pseudoscalar screening masses are significantly smaller. This observation, and the subsequent measurement [5] of transverse spatial correlations for distances up to roughly $2 / T$, require understanding.

We examine a generalised susceptibility, i.e., a propagator at zero 4-momentum. This is intimately connected with many details of the low-lying structure of the spectral function. We find that, for $T<T_{c}$ in the pseudoscalar meson channel, it is dominated by 
a bosonic pole. However, for $T>T_{c}$, there is no evidence of such a pole; a multifermion cut is indicated by the data. Thus, in all channels, the leading contribution to the spectral density comes from unbound quarks, which turn out to have fairly strong pseudoscalar effective self-interactions close to $T_{c}$.

We restrict ourselves to a quenched QCD computation. In the quenched theory, a measurement of the chiral condensate, $\langle\bar{\psi} \psi\rangle$, in the limit $m_{q}=0$, shows a discontinuity at the deconfinement transition. Furthermore, in the quenched theory, spatial Wilson loops continue to display an area law behaviour above $T_{c}$ [8]. Thus all the phenomena of interest in full QCD are present in some form in the quenched theory. Finally, because of the relatively low CPU requirements, the extrapolation to zero quark mass and finite spatial-size effects can be studied in detail. Nevertheless, we look upon this as an exploratory study, whose conclusions must be checked through simulations of QCD with dynamical fermions.

Correlation functions and susceptibilities: Consider the gauge-invariant mesonic correlator

$$
G_{\Gamma}(x-y)=i\left\langle M_{\Gamma}(x) M_{\Gamma}^{\dagger}(y)\right\rangle
$$

where the expectation value is measured over equilibrium configurations on a lattice of temporal extent $N_{\tau}$ and lattice spacing $a\left(T=a / N_{\tau}\right)$. The operator $M_{\Gamma}$ may be constructed as a local fermion-bilinear current

$$
M_{\Gamma}(x)=\bar{\psi}(x) \Gamma \psi(x)
$$

where $\Gamma$ denotes some combination of Dirac and flavour matrices. We shall identify channels by the Dirac content, and use the notation $\sigma$ for $\Gamma=1, \pi$ for $\gamma_{5}, \rho$ for $\gamma_{\mu}$ and $A$ for $\gamma_{5} \gamma_{\mu}$. The long-distance decay of a spatial correlation function determines the screening mass $\mu$ in that channel.

For a finite-temperature computation in the imaginary-time formalism, $G_{\Gamma}$ can be represented as the Fourier transform of the momentum-space correlator

$$
G_{\Gamma}(x)=T \sum_{n} \mathrm{e}^{i \omega_{n} x_{0}} \int \frac{d^{3} p}{(2 \pi)^{3}} \mathrm{e}^{i \mathbf{p} \cdot \mathbf{x}} G_{\Gamma}\left(\omega_{n}, p\right) .
$$

Since all boson fields are periodic in the $x_{0}$ direction, $\omega_{n}$ takes on the values $2 n \pi i T$ $(n=0, \pm 1, \cdots)$. For fermion fields antiperiodicity implies that $\omega_{n}$ takes values in $(2 n+1) \pi i T$. The propagator has the spectral representation

$$
G_{\Gamma}\left(\omega_{n}, p\right)=i \int \frac{d p_{0}}{2 \pi} \frac{\rho_{\Gamma}\left(p_{0}, p\right)}{\omega_{n}-p_{0}}
$$

where $\rho_{\Gamma}(p)$ is the spectral density in the given channel [9]. The spectral function allows us to identify the physical degrees of freedom in a plasma. 
This spectral representation allows us to rewrite the (spatial) 3-momentum projected temporal correlator as the contour integral

$$
G_{\Gamma}(\tau, \mathbf{p})=\frac{1}{2 \pi i} \oint d p_{0} \rho_{\Gamma}\left(p_{0}, \mathbf{p}\right) \frac{\mathrm{e}^{-p_{0} \tau}}{1-\mathrm{e}^{-p_{0} / T}}
$$

The contour avoids all the poles of the Bose function $1 /\left(\exp \left(p_{0} / T\right)-1\right)$ and includes all the singularities of $\rho(p)$. A similar contour representation of the momentum-projected spatial correlators can also be written. These can be easily transcribed to a lattice with periodic boundary conditions in the spatial directions.

Apart from the spatial correlators, we shall also consider generalised susceptibilities. These are defined in the usual way by adding to the action a source term coupling to a current of the correct quantum numbers, and then taking two derivatives of the partition function with respect to this source. A fluctuation-dissipation theorem equates $\chi_{\Gamma}$ to the meson propagator at zero 4-momentum

$$
\chi_{\Gamma}=G_{\Gamma}(p=0)
$$

$\chi$ may be obtained as a sum over all separations of the zero-(3)momentum spatial correlator.

The physical degrees of freedom: The spectral function $\rho_{\Gamma}$ has a pole for each physical degree of freedom with quantum numbers $\Gamma$. It also has a branch cut for every allowed multiparticle state carrying the same quantum numbers. The screening mass obtained from the spatial zero-(3)momentum correlator depends on the singularity closest to the origin. If this is a pole, then $\chi_{\Gamma}$ diverges as $1 / \mu^{2}$ when $\mu$ is extrapolated to zero, i.e., as the point $p^{2}=0$ moves on-shell. Finite-size effects in such an extrapolation can be understood by studying the origin of this behaviour. Substituting a pole model into Eq. (4) and constructing the spatial correlator, we find that one factor of $\mu$ arises from the normalisation. The zero-momentum projection yields the second factor. This allows us to compute whether the lattice is long enough for a given $\mu$. No substantial change occurs due to a distant cut in $\rho_{\Gamma}$. However, if the lip of the cut approaches and overlaps the pole, then the power-law for the diveregence is changed into a non-trivial critical index.

It is interesting to see how these considerations are enforced at zero temperature in QCD. A Ward identity relates $\chi_{\pi}$ to $\langle\bar{\psi} \psi\rangle[10]$

$$
m_{q} \chi_{\pi}=\langle\bar{\psi} \psi\rangle
$$

The Gell-Mann-Oakes-Renner identity,

$$
m_{\pi}^{2} f_{\pi}^{2}=m_{q}\langle\bar{\psi} \psi\rangle
$$


taken in conjunction with this, implies a $1 / m_{\pi}^{2}$ variation of the susceptibility. Thus, at zero temperature, Eqs. 7 and 8 guarantee the presence of a pion pole in the spectral density, and enforce the Goldstone theorem by forcing the position of this pole to zero. Conversely, for $0<T<T_{c}$, the Goldstone theorem forces a pion pole, and relates $f_{\pi}$ to the residue, i.e., the renormalisation constant. In the chiral limit of the hot phase, these identities are trivially satisfied with $\langle\bar{\psi} \psi\rangle=f_{\pi}=m_{q}=0$. Thus the existence of a pole is no longer guaranteed.

The spatial correlator yields a screening mass even in a theory of non-interacting quarks. This arises from a cut starting at the Matsubara frequency [11]. On the lattice a cut is a discrete series of masses [4], the lowest of which is

$$
\mu_{0} a=2 \sinh ^{-1}\left(\sqrt{a^{2} m_{q}^{2}+\sin ^{2} \pi / N_{\tau}}\right) .
$$

Such a cut has been identified in the $\rho$ and $A$ channels, already very close to $T_{c}$, thus demonstrating that quarks exist as physical degrees of freedom in a plasma ${ }^{1}$.

The fact that the screening mass in the $\pi$ and $\sigma$ channels, close to $T_{c}$, is smaller than $\mu_{0}$ can have several interpretations. The simplest is that in this channel there is a pole [12]. It has also been suggested [13] that a pole coexists with a cut, as in a $\sigma$-model. It is also possible that there is only a cut, but it is due to interacting fermions. In such a scenario the screening mass still arises from the lip of the cut. It is interesting to note that such a measurement can then be made to yield a phase shift, in a fermionic analogue of the arguments developed in detail in [14]. In a Minkowski theory, of course, a cut and a pole can be distinguished by looking at the imaginary part of the correlation function. Here, the susceptibility allows us to make this distinction. For a cut, and no lower-lying pole, $\chi_{\Gamma}$ goes to a non-zero constant as $\mu$ is extrapolated to zero. This constant is a renormalised coupling.

The simulations: The simulations were performed at $\beta=5.5,5.75,5.8941$, and 6.05 on lattices of size $N_{\tau} \times N_{r}^{2} \times N_{z}\left(N_{\tau}=4\right)$. The critical coupling in the thermodynamic limit is estimated to be 5.6922 [15]. From the results of [16] we find that $\beta=5.8941$ corresponds to $1.5 T_{c}$, and $\beta=6.05$ to $2 T_{c}$. The lowest coupling, $\beta=5.5$, corresponds to $T \simeq 0.7 T_{c}$, and has been used in an earlier computation of hadron masses [17] at zero temperature, on a lattice of spatial size similar to our smallest. Configurations were generated by a pseudo-heat bath algorithm [18]. Some details of the runs are given in Table 1.

1 This implies that the matrix elements for photon and dilepton production rates in a plasma can now be inferred from a lattice computation. 


\section{TABLE 1}

We list some details of our simulations and measurements. The lattice sizes are $N_{\tau} \times N_{r}^{2} \times N_{z}$, the couplings $\beta$, the number of configurations for the measurement of hadronic correlation functions $N_{\text {conf }}$, and the spatial and temporal plaquette values $P_{s}$ and $P_{t}$. Stored configurations are separated by 500 sweeps. The first 10 have been discarded for thermalisation in each case.

\begin{tabular}{|c|l|r|c|c|}
\hline Lattice & \multicolumn{1}{|c|}{$\beta$} & $N_{\text {conf }}$ & $P_{s}$ & $P_{t}$ \\
\hline $4 \times 8^{2} \times 16$ & 5.5 & 70 & $0.49683(9)$ & $0.49688(9)$ \\
& 5.75 & 140 & $0.56277(4)$ & $0.56408(5)$ \\
& 5.8941 & 70 & $0.58227(5)$ & $0.58382(4)$ \\
& 6.05 & 70 & $0.59921(4)$ & $0.60078(4)$ \\
\hline $4 \times 8^{2} \times 32$ & 5.5 & 80 & $0.49678(5)$ & $0.49683(5)$ \\
\hline $4 \times 12^{2} \times 24$ & 5.75 & 80 & $0.56281(3)$ & $0.56410(3)$ \\
& 6.05 & 80 & $0.59920(2)$ & $0.60080(2)$ \\
\hline $4 \times 16^{2} \times 32$ & 5.75 & 80 & $0.56285(2)$ & $0.56412(2)$ \\
& 6.05 & 80 & $0.59919(1)$ & $0.60080(1)$ \\
\hline
\end{tabular}

For the screening masses, the standard practice $[3,4]$ is to construct the correlation functions

$$
G_{C}(z)=\sum_{x, y, \tau} \alpha_{C}(x, y, \tau)\langle\bar{\chi}(x) \chi(0)\rangle .
$$

The phase factors are $\alpha_{P S}=1, \alpha_{V T}=(-1)^{x}+(-1)^{y}+(-1)^{\tau}$, and have to be multiplied by $(-1)^{x+y+\tau}$ for the parity-reversed channels $S$ and $P V$ respectively. We constructed spin-projected correlation functions [19] from the linear combination

$$
G_{\Gamma}(2 z)=\frac{1}{2}\left[G_{C}(2 z+1)+2 G_{C}(z)+G_{C}(2 z-1)\right] .
$$

The combinations we need are identified by the $(\Gamma, C)$ pairs $(\sigma, S),(\pi, P S),(\rho, V T)$ and $(A, P V)$. These are defined on half the lattice points. The susceptibilities are the sum over all allowed lattice points of the appropriate correlator.

The fermion matrix was inverted with quark masses $0.01 \leq m_{q} \leq 0.15$, using a conjugate gradient algorithm with a stopping criterion of $m_{q}^{2} 10^{-8} / 4$. We have checked that sharpening this by two orders of magnitude does not change the pion propagator. We extracted masses from the spin-projected correlators through two- and four-parameter fits of the form

$$
G_{\Gamma}(z)=A_{\Gamma}^{1} \cosh \left[m_{\Gamma}^{1}\left(N_{z} / 2-z\right)\right]+A_{\Gamma}^{2} \cosh \left[m_{\Gamma}^{2}\left(N_{z} / 2-z\right)\right],
$$

and an off-diagonal $\chi^{2}$ definition. We found this procedure more congenial than trying four-parameter fits to the $V T, P V$ and $S$ correlators, specially since covariances between 
fitted masses could be completely eliminated by this means. When possible, the smallest mass was isolated by performing fits for $2 z \geq 2 z_{m i n}$, and changing $2 z_{m i n}$ until a stable estimate was obtained. This measurement was then validated by a four-parameter fit. On our smallest lattice, $4 \times 8^{2} \times 16, N_{z}$ was too short to perform such an analysis.

Screening masses for $T<T_{c}$ : At $\beta=5.5$, for equal values of $m_{q}, \mu_{\pi}$ is not distinguishable from the zero-temperature mass [17]. The $m_{q}=0$ extrapolation of the fits yields

$$
\mu_{\pi}^{2} a^{2}= \begin{cases}(0.008 \pm 0.005)+(6.6 \pm 0.2) m_{q} a & \left(N_{z}=16\right) \\ (0.005 \pm 0.002)+(6.60 \pm 0.04) m_{q} a & \left(N_{z}=32\right)\end{cases}
$$

Chiral symmetry remains broken, as is confirmed by the $m_{q}=0$ extrapolation

$$
\langle\bar{\psi} \psi\rangle a^{3}= \begin{cases}0.227 \pm 0.006 & \left(N_{z}=16\right) \\ 0.231 \pm 0.005 & \left(N_{z}=32\right)\end{cases}
$$

Assuming that the Gell-Mann-Oakes-Renner identity holds also at finite temperature, we can extract the value of $f_{\pi}$ using the measured value of $\langle\bar{\psi} \psi\rangle$ and the slope $m_{\pi}^{2} / m_{q}$ obtained from the fits above. We find

$$
f_{\pi} a= \begin{cases}0.185 \pm 0.007 & \left(N_{z}=16\right) \\ 0.187 \pm 0.002 & \left(N_{z}=32\right)\end{cases}
$$

This is a little smaller than the value in [17].

We have been unable to get a useful signal for the $\sigma$ and $A$ masses. The $\rho$ correlator also turned out to be noisy. We were able to extract a signal only for $m_{q} a=0.1$ and $2 z \leq 8$. Our best estimate for the mass is

$$
\mu_{\rho} a= \begin{cases}1.4 \pm 0.1 & \left(N_{z}=16\right) \\ 1.47 \pm 0.02 & \left(N_{z}=32\right)\end{cases}
$$

This is somewhat higher the value reported in [17]. Although this could signal a thermal mass shift in the $\rho$, it is necessary to significantly improve the measurement before any definite statement can be made.

Screening masses for $T>T_{c}$ : Mass measurements for $T>T_{c}$ turn out to be considerably easier. The correlation functions were much less noisy. We were able to follow all the correlation functions right to the centre of the lattice. At $1.5 T_{c}$ and $2 T_{c}$ we found negligible finite-size effects in the screening masses on all the lattices. In contrast, the effect was significant at $\beta=5.75$, on moving from the $4 \times 8^{2} \times 16$ to the $4 \times 12^{2} \times 24$ lattice, but again small in going to the $4 \times 16^{2} \times 32$ lattice. This is readily understood. Recall that the transition is first-order. It has been observed [15] that $\beta=5.75$ lies well within the region of metastabilities on the smallest lattice, but not on the others. The 
higher values of $\beta$ lie well away from this region on all the lattices. Thus the finite-size effects, as in most first-order transitions, are largely dominated by flips.

It was possible to extract masses in all channels with good confidence, and the $m_{q}$-dependence of all the screening masses could be investigated with greater precision. The linear extrapolation of masses with $m_{q}$ turned out to be good. It is interesting that at $\beta=5.75$ on the $4 \times 8^{2} \times 16$ lattice, neither an extrapolation of $m_{\pi}$ nor one of $m_{\pi}^{2}$ is statistically valid. This is what one expects from metastabilities between chiral symmetric and broken phases. In agreement with previous measurements $[3,4], \mu_{\rho}$ and $\mu_{A}$ are compatible with $\mu_{0}$ immediately above $T_{c}$. Furthermore, as $T$ increases, $\mu_{\pi}$ as well as $\mu_{\sigma}$ approach the same limit. Our best estimates for the screening masses are shown in Table 2.

\section{TABLE 2}

Estimates for the screening masses in the limit $m_{q}=0$ for $T>T_{c}$ from the lattices on which finite-size effects can be neglected. A free fermion theory would yield screening masses $\mu_{0} a=1.32$ in all channels.

\begin{tabular}{|l|l|c|c|c|}
\hline \multicolumn{1}{|c|}{$\beta$} & \multicolumn{1}{|c|}{$\mu_{\pi} a$} & $\mu_{\sigma} a$ & $\mu_{\rho} a$ & $\mu_{A} a$ \\
\hline 5.75 & $0.786 \pm 0.002$ & $0.847 \pm 0.007$ & $1.34 \pm 0.01$ & $1.32 \pm 0.05$ \\
5.8941 & $1.05 \pm 0.01$ & $1.08 \pm 0.01$ & $1.33 \pm 0.03$ & $1.37 \pm 0.05$ \\
6.05 & $1.13 \pm 0.01$ & $1.14 \pm 0.02$ & $1.36 \pm 0.02$ & $1.38 \pm 0.04$ \\
\hline
\end{tabular}

Susceptibilities: We have obtained the susceptibilities as explained earlier. $\chi_{\rho}$ is compatible with the free fermion value. $\chi_{\pi}$ has a strong dependence on $\beta$, and approaches the free value at $T=2 T_{c}$. However, close to and below $T_{c}$ it is significantly different.

Below $T_{c}$, at $\beta=5.5$, there is a physical pion state giving rise to a pole in the spectral function. On the lattice this manifests itself as a $1 / \mu \sinh \mu$ dependence of $\chi$ on the measured screening mass. In fact, a fit of the form $1 / 2 \chi=A \mu \sinh \mu+B$ yields

$$
B= \begin{cases}0.0008 \pm 0.0004 & \left(4 \times 8^{2} \times 16 \text { lattice }\right) \\ 0.0008 \pm 0.0003 & \left(4 \times 8^{2} \times 32 \text { lattice }\right)\end{cases}
$$

Finite-size effects are invisible, being entirely within the error bars.

Above $T_{c}$ the behaviour of this susceptibility is completely different. A fit of the form above is still possible, but the value of $B$ is now different from zero. At $\beta=5.75$ we find

$$
B= \begin{cases}0.041 \pm 0.003 & \left(4 \times 12^{2} \times 24 \text { lattice }\right) \\ 0.043 \pm 0.004 & \left(4 \times 16^{2} \times 32 \text { lattice }\right)\end{cases}
$$


Thus, the presence of a pion pole is ruled out by $10 \sigma$. Finite-size effects are strong on our smallest lattice, but not on the two larger volumes quoted above. The data for $\chi_{\pi}$ at $\beta=5.5$ and 5.75 are shown in Fig. 1 .

The absence of a pole in the lightest channel indicates that the only stable hadronic degrees of freedom are unbound, but perhaps interacting, quarks. Hence, one can construct several measures of the interaction strength between quarks. We report the dimensionless ratio

$$
g_{\Gamma} T^{2}=\frac{N_{\tau}^{2}}{2}\left(\frac{1}{\chi_{\Gamma}^{(0)}}-\frac{1}{\chi_{\Gamma}}\right)
$$

where $\chi_{\Gamma}^{(0)}$ denotes the susceptibility computed with non-interacting fermions on a lattice of equal size. This gives an effective temperature-dependent 4 -fermi coupling, as can be verified by summing the "ladder" diagrams for $\chi$ in an effective 4-fermi theory. We discuss the perturbative evaluation of this effective coupling later.

Our results are collected in Table 3 . We find it interesting to note that the effective coupling decreases extremely fast with the temperature. The coupling in the vector channel becomes consistent with zero at $1.5 T_{c}$, i.e., $\chi_{\rho}$ becomes compatible with that due to non-interacting quarks. However, even at $2 T_{c}$, the coupling in the pseudoscalar channel remains stronger than the vector channel coupling just above $T_{c}$.

\section{TABLE 3}

The effective 4-fermi couplings in the vector and pseudoscalar channels on different lattice sizes and varying $\beta$, after extrapolation to $m_{q}=0$.

\begin{tabular}{|c|l|c|r|}
\hline Lattice & \multicolumn{1}{|c|}{$\beta$} & $g_{\gamma_{5}} T^{2}$ & \multicolumn{1}{|c|}{$g_{\gamma_{\mu}} T^{2}$} \\
\hline $4 \times 8^{2} \times 16$ & 5.75 & $1.19 \pm 0.03$ & $0.21 \pm 0.03$ \\
& 5.8941 & $0.61 \pm 0.04$ & $0.03 \pm 0.04$ \\
& 6.05 & $0.38 \pm 0.02$ & $-0.02 \pm 0.03$ \\
\hline $4 \times 12^{2} \times 24$ & 5.75 & $1.10 \pm 0.05$ & $0.14 \pm 0.04$ \\
& 6.05 & $0.45 \pm 0.02$ & $0.03 \pm 0.03$ \\
\hline $4 \times 16^{2} \times 32$ & 5.75 & $1.19 \pm 0.06$ & $0.21 \pm 0.05$ \\
& 6.05 & $0.39 \pm 0.03$ & $-0.03 \pm 0.03$ \\
\hline
\end{tabular}

A perturbative estimate: An explicit computation to order $g^{2}$ in perturbation theory shows a stronger effect in the $\sigma / \pi$ than in the $\rho / A$ channels. The diagrams contributing to $\chi$ up to this order are given in Fig. 2. With the notation

$$
\chi_{\Gamma}=N_{c} \chi_{\Gamma}^{(0)}\left(1+g^{2} r_{\Gamma}^{(1)}\right)
$$


$\chi_{\Gamma}^{(0)}$ and $\chi_{\Gamma}^{(0)} r_{\Gamma}^{(1)}$ can be written down by inspection of the diagrams. Parity-related channels have equal and opposite values to all orders in $g^{2}$. We present the results of a computation with naive fermions. We evaluate the graphs, do the Dirac algebra and obtain a sum over lattice momenta. The leading-order result coincides with that obtained using staggered fermions. Evaluating the sums numerically, we find that the result stabilises at fairly small volumes for any given $N_{\tau}$. On a $4 \times 16^{2} \times 32$ lattice, we find $r_{\rho}^{(1)}=0.1171$, whereas $r_{\pi}^{(1)}=0.7699$. This large correction argues for a computation directly with staggered fermions, and a resummation. The analysis can, in fact, be extended to the full spatial correlator, and hence the screening mass. For this, it is sufficient to analyse every 4 -momentum component $G(k)$ separately. The perturbative corrections are due to the same diagrams as before, with a net flow of momentum $k$ through the fermion loop.

Conclusions: We have performed an analysis of meson correlation functions in finite-temperature quenched QCD. For $T<T_{c}$, the pseudoscalar susceptibility signals a bosonic pole in the spectral density. There is some evidence of a finite-temperature shift in the mass of the vector, but a more detailed study is necessary to identify this properly. Above $T_{c}$, the lowest mass in the vector/pseudovector channels is equal to twice the Matsubara frequency, and thus compatible with weakly interacting fermions. The smaller screening mass for the scalar/pseudoscalar was investigated through the susceptibility. The scaling of the susceptibility indicates that there is no pole of the spectral density corresponding to a pion. These measurements demonstrate that information on the spectral density can be extracted from lattice measurements. The screening mass in the pseudoscalar channel gives evidence for a strong interquark interaction in this channel. Our analysis reveals that this information is isolated in the susceptibility. Detailed studies of the cut in the scalar and pseudoscalar channels yield information on interactions in the plasma. It is amusing to note the similarity between these measurements and the dispersion relations approach to strong interactions at zero temperatures.

Because of the large lattice spacing, it is entirely possible that we have missed out interesting structures in the spectral function close to the origin. Computations with larger values of $N_{\tau}$ are necessary to remedy this. Similarly, the structure of the singularities in QCD with dynamical fermions needs investigation using an extension of these techniques. We believe that such a program must be pursued in order to make contact with forthcoming experiments.

Acknowledgements: The simulations have been performed on the Cray-XMP at CERN. I would like to thank H. R. Renshall and E. McIntosh of the CN division for their active support. I would also like to thank Anders Irbäck and Rainer Sommer for 
interesting and useful discussions.

\section{REFERENCES}

1) D. J. Gross, R. D. Pisarski and L. G. Yaffe, Rev. Mod. Phys. 53 (1981) 43.

2) J. Engels, J. Fingeberg, F. Karsch, D. Miller and M. Weber, Phys. Lett. B252 (1990) 625.

3) C. Detar and J. Kogut, Phys. Rev. Lett. 59 (1987) 399; Phys. Rev. D36 (1987) 2828 ;

S. Gottlieb, W. Liu, R. L. Renken, R. L. Sugar, and D. Touissant Phys. Rev. Lett. 59 (1987) 1881;

A. Gocksch, P. Rossi and U. M. Heller, Phys. Lett. B205 (1988) 334.

4) K. Born, S. Gupta, A. Irbäck, F. Karsch, E. Laermann, B. Petersson and H. Satz, Phys. Rev. Lett. 67 (1991) 302.

5) C. Bernard, M. C. Ogilvie, T. DeGrand, C. DeTar, S. Gottlieb, A. Krasnitz,

R. Sugar and D. Touissant, preprint AZPH-TH/91-60.

6) G. Boyd, S. Gupta and F. Karsch, preprint BI-TP 92-15.

7) S. Gottlieb, W. Liu, D. Touissant, R. L. Renken, R. L. Sugar, Phys. Rev. Lett. 59 (1987) 2247;

R. V. Gavai, J. Potvin and S. Sanielevici, Phys. Rev. D40 (1989) 2743.

8) E. Manousakis and J. Polonyi, Phys. Rev. Lett. 58 (1987) 847.

9) L. Dolan and R. Jackiw, Phys. Rev. D9 (1974) 3320.

10) J. Kogut, M. Stone, H. W. Wyld, S. H. Shenker, J. Shigemitsu and D. K. Sinclair, Nucl. Phys. B225 (1983) 326;

G. W. Kilcup and S. R. Sharpe, Nucl. Phys. B283 (1987) 493.

11) V. L. Eletskii and B. L. Ioffe, Sov. J. Nucl. Phys. 48 (1988) 384.

12) C. DeTar, Phys. Rev. D32 (1985) 276; Phys. Rev. D37 (1987) 2328.

13) A. Gocksch, Phys. Rev. Lett. 67 (1991) 1701.

14) M. Lüscher, Nucl. Phys. B354 (1991) 531.

15) M. Fukugita, M. Okawa and A. Ukawa, Nucl. Phys. B337 (1990) 181.

16) Y. Iwasaki et al., Phys. Rev. Lett. 67 (1991) 3343.

17) M. Fukugita, S. Ohta, Y. Oyanagi and A. Ukawa, Phys. Lett. 191B (1987) 164.

18) N. Cabibbo and E. Marinari, Phys. Lett. 119B (1982) 387.

19) H. Kluberg-Stern, A. Morel, O. Napoli and B. Petersson, Nucl. Phys. B220 (1983) 447 ;

J. P. Gilchrist, G. Schierholz, H. Schneider and M. Teper, Nucl. Phys. B248 (1984) 29. 


\section{FIGURE CAPTIONS}

Fig. 1: $\chi_{\pi}$ as a function of the pion screening mass at (a) $\beta=5.5$ and (b) $\beta=5.75$. A zero intercept shows the presence of a pion pole. Finite size effects are seen to be under control.

Fig. 2: The perturbative diagrams contributing in the quenched theory to $\chi_{\Gamma}$ at (a) lowest order, and (b, c, d) order $g^{2}$. 


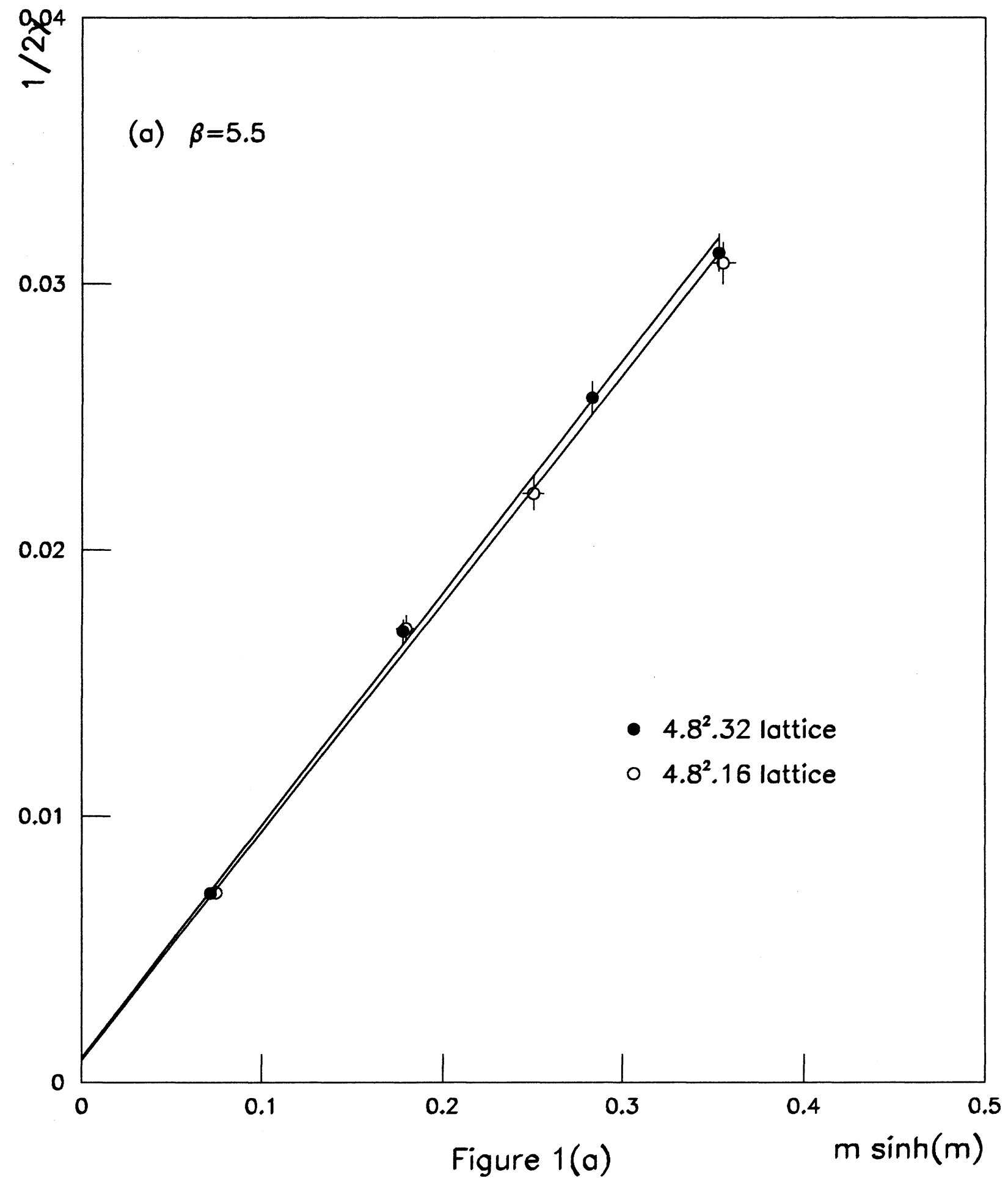




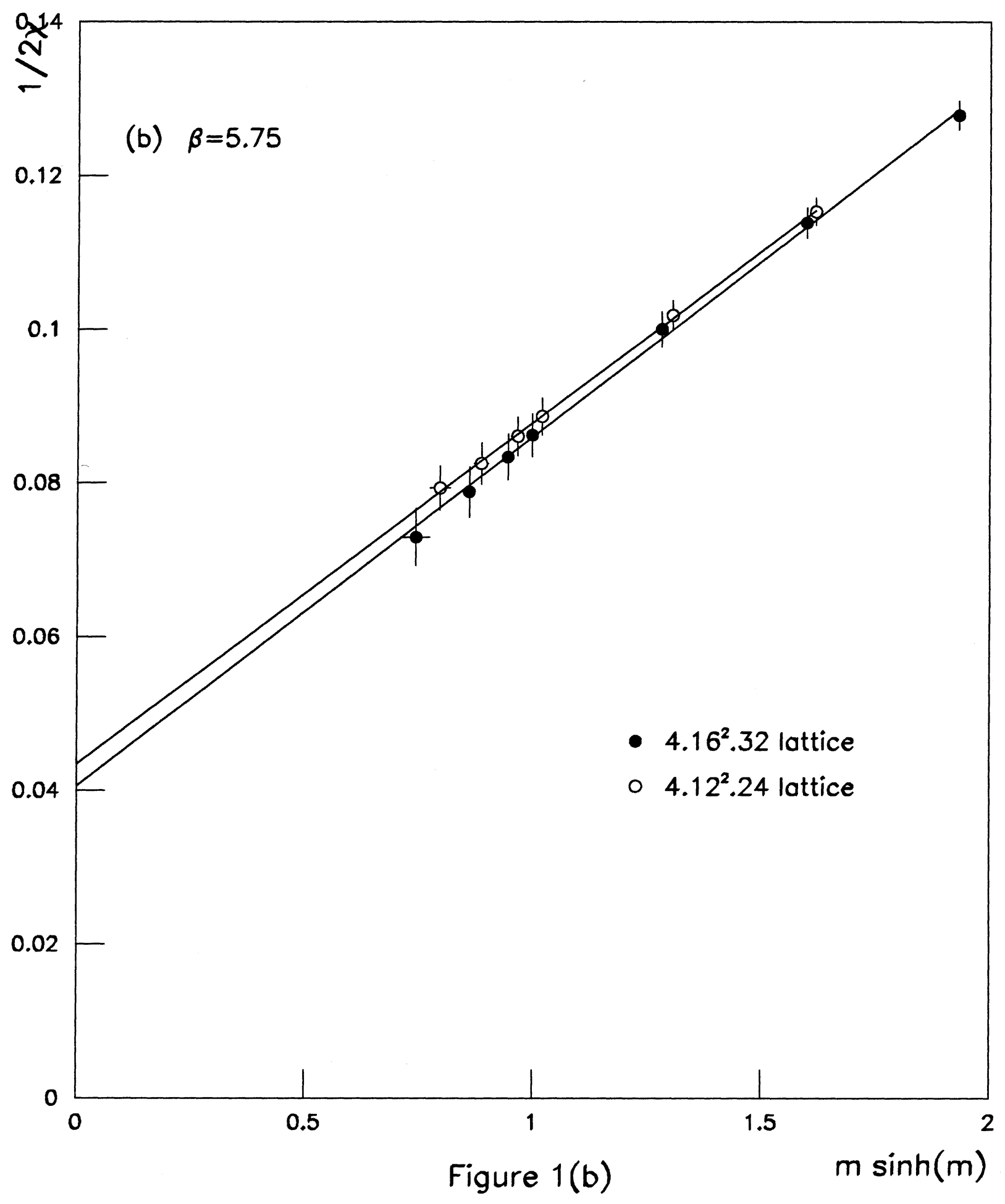




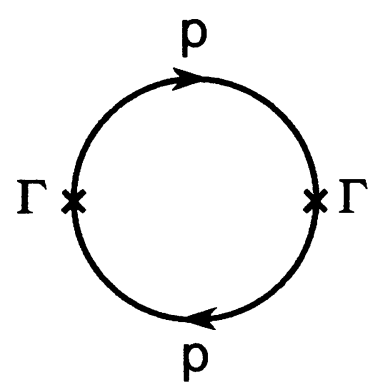

(a)

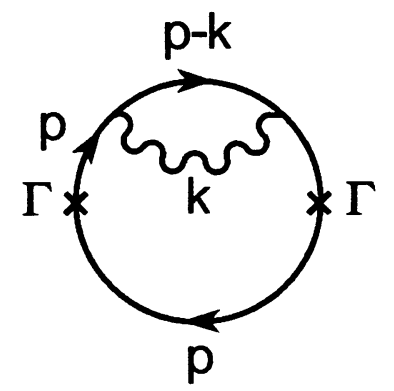

(b)

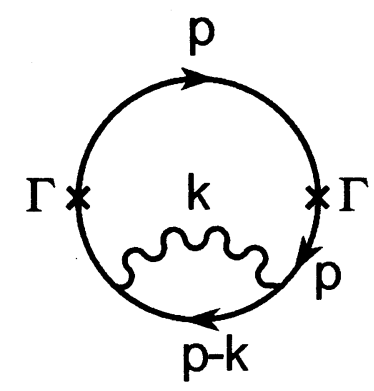

(c)

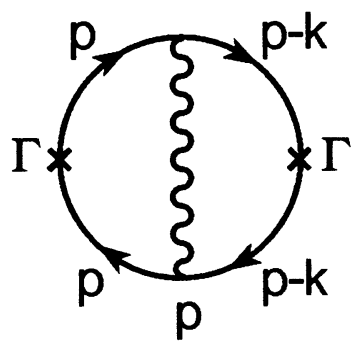

(d) 